\title{
CAPACIDADE DE ESTABILIZAÇÃO PÉLVICA EM NADADORES DE DIFERENTES ESTILOS
}

\author{
PELVIC STABILIZATION CAPACITY IN DIFFERENT STYLES SWIMMERS
}

Artigo Original

CAPACIDAD DE ESTABILIZACIÓN PÉLVICA EN NADADORES DE DIFERENTES ESTILOS

OrIGINAL ARTICLE

Artículo Original
Juliana Alves de Andrade ${ }^{1}$ (Fisioterapeuta)

Thiago Ribeiro Teles dos Santos ${ }^{1}$ (Fisioterapeuta)

Luciano Sales Prado²

(Educador Físico)

Sérgio Teixeira da Fonseca

(Fisioterapeuta)

1. Departamento de Fisioterapia da Escola de Educação Física,

Fisioterapia e Terapia Ocupacional, Universidade Federal de Minas Gerais, Belo Horizonte, MG, Brasil. 2. Departamento de Educação

Física da Escola de Educação Física, Fisioterapia e Terapia Ocupacional, Universidade Federal de Minas Gerais, Belo Horizonte, MG, Brasil.

\section{Correspondência:}

Sérgio Teixeira da Fonseca. Laboratório de Prevenção e Reabilitação de Lesões Esportivas, Centro de Excelência Esportiva, Escola de Educação Física, Fisioterapia e Terapia Ocupacional, Universidade Federal de Minas Gerais. Av. Presidente Antônio Carlos, 6627, 31270-901, Belo Horizonte, MG, Brasil. sfonseca@pib.com.br

\section{RESUMO}

Introdução: os estilos de nado com movimentos alternados ou simultâneos dos membros superiores podem gerar demandas diferentes sobre os músculos lombopélvicos. A avaliação do alinhamento pélvico no plano transverso contribui para a compreensão da influência dessas diferentes demandas decorrentes do nado sobre a estabilidade pélvica. Objetivos: investigar o efeito do treinamento de estilos com braçadas alternadas ou com braçadas simultâneas sobre a estabilização pélvica no plano transverso. Métodos: foram avaliados 113 nadadores, 63 praticantes de braçada alternada e 50 de braçada simultânea, por meio do teste da ponte com extensão unilateral do joelho. A magnitude e assimetria de queda pélvica foram quantificadas por meio de programa de análise de movimento. O teste de Mann-Whitney foi utilizado para verificar diferenças na assimetria de queda pélvica entre atletas que praticavam braçada alternada e simultânea em cada repetição do teste. A ANOVA com desenho misto foi realizada para investigar diferenças na magnitude de queda pélvica entre repetições considerando a braçada praticada. Resultados: a assimetria de queda pélvica não apresentou diferença entre grupos em nenhuma repetição. A magnitude de queda pélvica foi maior na terceira repetição $\left(15,96 \pm 7,1^{\circ}\right)$ do que na primeira $\left(15,13 \pm 7,52^{\circ}\right)$ e segunda $\left(15,16 \pm 7,35^{\circ}\right)$ em todos os nadadores. Os praticantes de braçada alternada apresentaram maior magnitude de queda pélvica do que os de braçada simultânea $\left(16,46 \pm 7,38^{\circ}\right.$ versus $\left.14,13 \pm 7,08^{\circ}\right)$. Conclusão: o tipo de braçada praticada não influencia a assimetria do alinhamento pélvico no plano transverso. Entretanto, a magnitude de queda pélvica foi maior na terceira repetição e em praticantes de braçada alternada.

Palavras-chave: pelve, natação, atletas.

\section{ABSTRACT}

Introduction: the swimming styles with alternate or simultaneous upper limb movements can generate different demands on the lumbopelvic muscles. The evaluation of pelvic alignment in transverse plane may contribute to the understanding of the influence of these demands arising from swimming on the pelvic stability. Objectives: to investigate the effect of alternated or simultaneous strokes on pelvic stabilization in the transverse plane. Methods: a total of 113 swimmers were evaluated, 63 performing alternated strokes and 50 simultaneous strokes, by means of the bridge test with unilateral knee extension. The magnitude and asymmetry of pelvic tilt (or drop) were quantified through motion analysis software. The Mann-Whitney test was used to assess differences in pelvic tilt asymmetry between athletes who practiced alternated and simultaneous stroke in each test repetition. Mixed-design ANOVA was performed to investigate differences in the magnitude pelvic tilt among repetitions according to the stroke practiced. Results: pelvic tilt asymmetry did not show differences between groups in any repetition. The magnitude of pelvic drop was higher in the third repetition $\left(15.96 \pm 7.18^{\circ}\right)$ than in the first $\left(15.13 \pm 7.52^{\circ}\right)$ and the second $\left(15.16 \pm 7.35^{\circ}\right)$ in all swimmers. The alternating stroke practitioners showed higher magnitude of pelvic tilt than simultaneous stroke ones $\left(16.46 \pm 7.38^{\circ}\right.$ versus $\left.14.13 \pm 7.08^{\circ}\right)$. Conclusion: the type of stroke practiced does not influence the asymmetry of pelvic alignment in the transverse plane. However, the magnitude of pelvic tilt was higher in the third repetition and in alternating stroke practitioners.

Keywords: pelvis, swimming, Athletes.

\section{RESUMEN}

Introducción: los estilos de nado con movimientos alternados o simultáneos de los miembros superiores pueden generar demandas diferentes sobre los músculos lumbopélvicos. La evaluación de la alineación pélvica en el plano transversal contribuye para la comprensión de la influencia de esas diferentes demandas provenientes del nado sobre la estabilidad pélvica. Objetivos: investigar el efecto del entrenamiento de estilos con brazadas alternadas o con brazadas simultáneas sobre la estabilización pélvica en el plano transversal. Métodos: fueron evaluados 113 nadadores, 63 practicantes de brazada alternada y 50 de brazada simultánea, a través del test del puente con extensión unilateral de la rodilla. la magnitud y asimetría de caída pélvica fueron cuantificadas por medio de programa de análisis de movimiento. El test de Mann-Whitney fue utilizado para verificar diferencias en la asimetría de caída pélvica entre atletas que practicaban brazada alternada y simultánea en cada repetición del test. Fue realizado ANOVA con diseño mixto para investigar diferencias en la magnitud de caída pélvica entre repeticiones considerando la brazada practicada. Resultados: La asimetría de caída pélvica no presentó diferencia entre grupos en ninguna repetición. La magnitud de caída pélvica fue mayor en la tercera repetición $\left(15,96 \pm 7,18{ }^{\circ}\right)$ que en la 
primera $\left(15,13 \pm 7,52^{\circ}\right)$ y segunda $\left(15,16 \pm 7,35^{\circ}\right)$ en todos los nadadores. Los practicantes de brazada alternada presentaron mayor magnitud de caída pélvica que los de brazada simultánea $\left(16,46 \pm 7,38^{\circ}\right.$ versus $\left.14,13 \pm 7,08^{\circ}\right)$. Conclusión: el tipo de brazada practicada no influencia la asimetría de la alineación pélvica en el plano transversal. Entretanto, la magnitud de caída pélvica fue mayor en la tercera repetición y en practicantes de brazada alternada.

Palabras clave: pelvis, natación, Atletas.

\section{INTRODUÇÃO}

A prática da natação impõe alta demanda à região lombopélvica dos atletas. Essa demanda pode ser entendida como quantidade de estresse (energia mecânica) imposta aos tecidos musculares e não musculares durante a prática esportiva ${ }^{1}$. A quantidade de estresse é induzida, entre outros fatores, pelos gestos esportivos da natação². Nessa perspectiva, diferentes estilos podem produzir adaptações teciduais distintas que, por sua vez, podem influenciar a capacidade de estabilização pélvica durante a natação. Por exemplo, os nados crawl e costas são caracterizados pelo movimento da pelve principalmente no plano transverso, enquanto que nos nados peito e borboleta, o movimento ocorre principalmente no plano sagital ${ }^{3}$. Dessa forma, a alta demanda imposta à região lombopélvica requer diferença na capacidade de estabilização pélvica de acordo com o nado praticado pelo atleta.

A capacidade de estabilização pélvica adequada permite a integração de segmentos corporais proximais e distais na transmissão e geração de força muscular e, consequentemente, contribuem para a realização dos gestos da natação ${ }^{4}$. A integração desses segmentos é associada à propulsão do atleta, na qual os movimentos realizados por membros superiores são responsáveis por mais de $70 \%$ da sua aceleração ${ }^{5}$. Alterações na estabilização pélvica podem resultar em redução do desempenho e em aumento no risco de lesões musculoesqueléticas do nadador ${ }^{4}$. Dessa forma, compreender como estilos diferentes do nado podem interferir na capacidade de estabilização pélvica permitiria o desenvolvimento de ações para prevenir lesões e aumentar o desempenho do atleta.

A integração de forças dos membros inferiores e superiores ocorre por meio de conexões da fáscia toracolombar com músculos que agem na região lombopélvica e nos membros superiores ${ }^{6-10}$. As forças transmitidas à cintura escapular contribuem para o posicionamento adequado da escápula durante a realização da braçada ${ }^{6,11}$. Isso permite que os músculos escapulares atuem de forma efetiva e assim, colaborem com a função do ombro na prática esportiva ${ }^{6,11}$. Da mesma forma, a força gerada por músculos do membro superior na braçada é transmitida à região lombopélvica por meio da escápula11. Portanto, a transmissão adequada de forças entre a região lombopélvica e escapular poderia proporcionar um melhor desempenho do atleta durante a braçada dos diversos nados.

Os diferentes estilos de nado geram demandas distintas sobre os músculos da região lombopélvica. Nos nados crawl e costas, o atleta realiza braçada e pernada alternadas associadas com rotação da pelve e do tronco em sentidos opostos ${ }^{12}$. Esse movimento no plano transverso permite minimizar posições que poderiam causar sobrecarga no ombro durante a braçada ${ }^{12}$. Estudos indicam que o aumento da amplitude de rotação do tronco durante os nados crawl e costas permite um melhor desempenho do atleta e reduz o desenvolvimento de lesões em membros superiores ${ }^{13}$. Por outro lado, nos nados peito e borboleta, o atleta realiza braçadas e pernadas simultâneas, sem movimento significativo da pelve e do tronco no plano transverso ${ }^{3}$. Nesses nados, a impulsão na água é realizada por ondulações no plano sagital acrescida de movimentos de membros superiores e inferiores ${ }^{14}$. Devido à grande demanda resultante da maior amplitude de movimento pélvico, a prática contínua dos nados crawl e costas, poderia resultar em maior capacidade de estabilização pélvica no plano transverso em relação ao treinamento prevalente dos nados peito e borboleta.

Os nados também poderiam influenciar a assimetria na capacidade de estabilização pélvica. Os nadadores de crawl e costas por realizarem maior amplitude de deslocamento pélvico no plano transverso poderiam estar mais susceptíveis a fatores que potencialmente geram assimetria. No nado crawl, por exemplo, a assimetria na capacidade de estabilização pélvica poderia estar relacionada ao movimento de tronco decorrente da rotação da cabeça para realizar a respiração ${ }^{3}$. A respiração unilateral pode levar à rotação excessiva do tronco e uso excessivo de um dos ombros $^{15}$. Além disso, alterações do alinhamento do membro inferior favorecem adaptações teciduais que geram assimetria na capacidade de estabilização pélvica. Por exemplo, atletas que apresentam discrepância no comprimento de membros inferiores apresentam o membro mais longo com adaptações teciduais que favorecem a rotação medial desse membro, gerando assim assimetria na estabilização pélvica ${ }^{16}$. Por outro lado, os praticantes dos nados peito e borboleta, por não executarem movimentos significativos no plano transverso, poderiam apresentar menor assimetria na capacidade de estabilização pélvica. Dessa forma, nadadores de crawl e costas poderiam apresentar maior assimetria na capacidade de estabilização pélvica que nadadores de peito e borboleta.

A avaliação da capacidade de estabilização pélvica no plano transverso contribui para a compreensão da influência dos estilos de nado na presença de desequilíbrios musculares e de alterações do alinhamento pélvico e assim, auxiliar na elaboração de programas de prevenção de lesões musculoesqueléticas ${ }^{4,17}$. O teste da ponte com extensão unilateral do joelho permite a avaliação da capacidade de estabilização pélvica no plano transverso por meio da mensuração do alinhamento pélvico nesse plano durante uma demanda do torque rotacional gerada pela elevação de um membro inferior ${ }^{18}$. Uma queda pélvica no plano transverso durante esse teste sugere baixo torque de resistência passiva e/ou ativa dos músculos lombopélvicos. Dessa forma, o objetivo deste estudo foi investigar o efeito do treinamento prevalente dos nados crawl e costas (estilos de nado com braçadas alternadas) ou peito e borboleta (estilos de nado com braçadas simultâneas) sobre a capacidade de estabilização pélvica no plano transverso.

\section{MÉTODOS}

Foram avaliados 113 atletas de natação, divididos em dois grupos de acordo com a especialidade de nado. $O$ primeiro grupo foi composto por 63 atletas que praticavam nados com braçada alternada (crawl ou costas) e o segundo, por 50 atletas que praticavam nados com braçada simultânea (borboleta ou peito). As características dos atletas estão apresentadas na tabela 1. Para participar deste estudo, o nadador deveria ter experiência mínima de cinco anos, a fim de que fossem selecionados atletas com adaptações teciduais decorrentes da prática esportiva². Os atletas também não deveriam apresentar dor lombar ou lesões musculoesqueléticas em membros inferiores. Além disso, três nadadores foram excluídos devido à câimbra ou dor durante a realização do teste, o que impossibilitou a sua continuidade. Os atletas e/ou responsável 
Tabela 1. Características dos nadadores por braçada praticada, apresentadas em média e desvio padrão.

\begin{tabular}{|c|c|c|c|}
\hline & \multicolumn{2}{|c|}{ Braçada } & \multirow[b]{2}{*}{$p$ valor } \\
\hline & $\begin{array}{c}\text { Alternada } \\
(\mathrm{N}=63)\end{array}$ & $\begin{array}{c}\text { Simultânea } \\
(\mathrm{N}=50)\end{array}$ & \\
\hline Idade (anos) & $14,74(1,84)$ & $14,82(2,00)$ & 0,98 \\
\hline Massa corporal $(\mathrm{kg})$ & $60,67(9,16)$ & $57,76(10,00)$ & 0,07 \\
\hline Estatura $(\mathrm{m})$ & $1,70(0,08)$ & $1,68(0,09)$ & 0,16 \\
\hline Tempo de prática esportiva (anos) & $9,25(4,08)$ & $9,35(4,33)$ & 0,97 \\
\hline Sexo, número de atletas do sexo masculino (\%) & $37(58,7)$ & $27(52,9)$ & 0,54 \\
\hline
\end{tabular}

(para nadadores menores de idade) assinaram o termo de consentimento livre e esclarecido. Este estudo foi aprovado pelo Comitê de Ética em Pesquisa da Instituição (parecer n ETIC 0493.0.203.000-09).

A especialidade do nado de cada atleta foi informada pela equipe técnica. A capacidade de estabilização pélvica no plano transverso foi avaliada por meio do teste da ponte com extensão unilateral do joelho ${ }^{19}$. Antes da realização desse teste, marcadores reflexivos de $10 \mathrm{~mm}$ de diâmetro foram colocados sobre as espinhas ilíacas anterossuperiores dos atletas, por um examinador previamente treinado. Esse procedimento tinha como objetivo facilitar a identificação dessas proeminências ósseas durante a análise do vídeo do teste. Para a realização do teste, o atleta foi posicionado em decúbito dorsal, as mãos colocadas sob a cabeça, com quadril e joelho fletidos em amplitude selecionada pelo próprio atleta, com as plantas dos pés próximas e apoiadas na maca ${ }^{19}$. O atleta foi orientado a elevar a pelve da maca e realizar a extensão de um dos joelhos, mantendo o membro inferior elevado na mesma altura que a coxa do membro contralateral, objetivando que tronco, quadril e membro inferior elevado ficassem posicionados em linha reta ${ }^{19}$ (figura 1). Antes da realização do teste, o atleta realizou o movimento uma vez com objetivo de familiarização ${ }^{19}$. Durante o teste, a posição foi sustentada por 10 segundos e esse procedimento foi realizado três vezes com cada membro inferior, alternadamente ${ }^{19}$. As orientações para execução do teste foram padronizadas e dadas por somente um examinador, previamente treinado.

O teste foi registrado com filmadora digital (SC-D385, Samsung ${ }^{\circledR}$, China) posicionada atrás da cabeça do participante, sobre um tripé a uma distância de $80 \mathrm{~cm}$ da extremidade da maca. A filmadora sobre o tripé era alinhada com inclinômetro (Mundo Sat, Brasil) de forma que ficasse paralela ao chão e sua altura, determinada de acordo com o plano de captação da pelve do participante, considerando assim, as características antropométricas de cada nadador.

A capacidade de estabilização pélvica no plano transverso foi operacionalizada como o alinhamento pélvico nesse plano durante o teste da ponte com extensão unilateral do joelho. Nessa operacionalização, um menor grau de queda pélvica no plano transverso é indicativo de boa capacidade de estabilização pélvica. Para determinar essa variável para cada membro inferior elevado realizou-se análise bidimensional do movimento utilizando o programa SIMI Motion Twin ${ }^{\circledR}$ (SIMI Reality Motion Systems, Alemanha). Nessa análise, inicialmente realizava-se uma calibração em uma distância de $30 \mathrm{~cm}$, a qual foi pré-determinada no ambiente de coleta ${ }^{19}$. O grau de queda pélvica no plano transverso foi mensurado por meio do ângulo formado por uma reta que passa no centro de cada marcador colocado sobre a espinha ilíaca anterossuperior e outra gerada pelo programa, que representa a horizontal no plano transverso pélvico ${ }^{19}$. A queda pélvica do atleta foi determinada pelo maior ângulo durante os 10 segundos, em que foi sustentado o membro inferior elevado ${ }^{19}$ (figura 1). A análise por meio desse programa apresentou confiabilidade intrateste excelente em estudo anterior (Coeficiente de Correlação Intraclasse - $\left.\mathrm{CCl}_{3,2}=0,82\right)^{19}$. Esse procedimento foi realizado por dois avaliadores que apresentaram confiabilidade intra e interobservador excelente $\left(\mathrm{CCl}_{3,2}=0,95\right.$ a 0,99) em estudo piloto.

A partir da análise do vídeo foi determinada a magnitude e assimetria

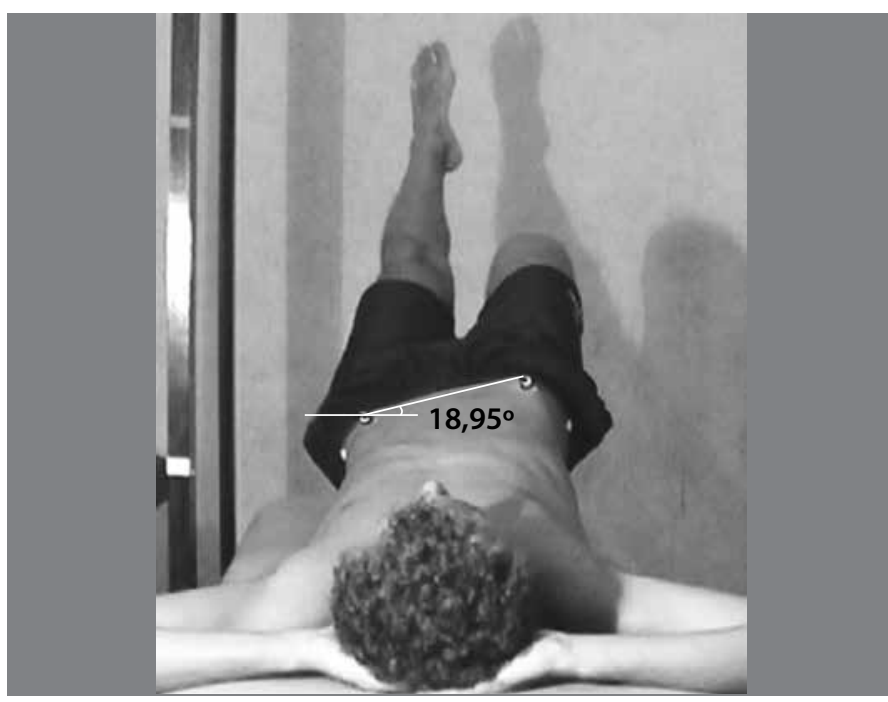

Figura 1. Avaliação do alinhamento pélvico no plano transverso durante o teste da ponte com extensão unilateral do joelho.

da queda pélvica em cada uma das três repetições do teste. A magnitude do ângulo de queda pélvica foi calculada para cada lado do corpo (tomando como referência o membro inferior elevado durante o teste). Para análise inferencial, a influência do nado na magnitude de queda pélvica foi investigada sem considerar a direção da queda (i.e. queda pélvica direita e esquerda). A assimetria foi determinada como a diferença entre os valores de magnitude para cada membro inferior elevado. Essa variável foi dada em valores absolutos, para que se considerasse o tamanho da assimetria e não sua origem (direita ou esquerda).

\section{Análise estatística}

Inicialmente, foi realizada a análise da distribuição das variáveis relacionadas às características da amostra (idade, peso, altura e tempo de prática esportiva) e das variáveis de desfecho (magnitude e assimetria de queda pélvica) por meio do teste de Kolmogorov-Smirnov e histogramas. Essa análise revelou que as variáveis relacionadas às características da amostra e magnitude de queda pélvica apresentavam distribuição normal, enquanto que a variável assimetria de queda pélvica apresentava distribuição não normal. Para verificar se havia diferença nas variáveis idade, peso, altura e tempo de prática esportiva entre os atletas que praticavam braçada alternada e simultânea foram realizados testes $t$ independentes. Para verificar se havia diferença entre a quantidade de homens e mulheres entre os dois grupos de atletas realizou-se o teste de qui-quadrado. Para verificar se havia diferença na assimetria de queda pélvica entre atletas que praticavam braçada alternada e simultânea realizou-se o teste de Mann-Whitney para cada repetição do teste. Os dados de assimetria foram expressos em mediana e os de magnitude, em média e desvio padrão de acordo com a repetição do teste e braçada praticada pelo atleta. Para verificar se havia diferença na magnitude de queda pélvica entre as repetições considerando a braçada que o atleta praticava, realizou-se o teste ANOVA com desenho misto (grupo e repetição). Na presença de efeito principal do fator repetição, contrastes com o ajuste de Bonferroni foram realizados a fim de verificar entre quais repetições se encontrava a diferença. $\mathrm{O}$ nível de significância (a) de 0,05 foi estabelecido para todos os testes.

\section{RESULTADOS}

A comparação das variáveis demográficas e tempo de prática esportiva não identificou diferença entre os grupos de atletas. Os dados descritivos dessas variáveis assim como os valores $p$ das comparações estão apresentados na tabela 1. 
O teste de Mann-Whitney não revelou diferença na assimetria de queda pélvica entre os atletas que praticam braçada alternada e simultânea na primeira $(U=1552,00, z=-0,31, p=0,76)$, segunda $(U=1427,00$, $z=-1,02, p=0,31)$ e terceira repetição $(U=1520,00, z=-0,49, p=0,62)$ do teste da ponte com extensão unilateral do joelho. Os valores da mediana de assimetria de queda pélvica entre os atletas que praticam braçada alternada e simultânea foram respectivamente 6,010 versus 6,030 na primeira, 6,080 versus $5,5^{\circ}$ na segunda e 6,32 ${ }^{\circ}$ versus $5,75^{\circ}$ na terceira repetição.

A ANOVA com desenho misto revelou um efeito principal significativo na repetição do teste da ponte com extensão unilateral do joelho na magnitude de queda pélvica $(F(2,448)=4,17, p=0,02)$. Além disso, foi observado um efeito principal significativo em relação à braçada praticada pelos atletas $(F(1,224)=6,77, p=0,01)$, no qual a queda pélvica foi maior nos atletas que praticam braçada alternada $\left(16,46^{\circ} \pm 7,38^{\circ}\right)$ do que nos praticantes de braçada simultânea $\left(14,13^{\circ} \pm 7,08^{\circ}\right)$. Não foi observada interação entre a repetição do teste e a braçada praticada pelos atletas $(F(2,448)=0,38, p=0,68)$. As análises de contrastes para as repetições revelaram que a terceira repetição $\left(15,96^{\circ} \pm 7,1^{\circ}\right)$ apresentou maior queda pélvica do que a primeira $\left(15,13^{\circ} \pm 7,52^{\circ}, p=0,02\right)$ e do que a segunda repetição $\left(15,16^{\circ} \pm 7,35^{\circ}, p=0,04\right)$. Não foi identificada diferença estatisticamente significativa entre a primeira e segunda repetição $(p=1,00)$.

\section{DISCUSSÃO}

Este estudo investigou a influência do estilo de nado (nados com braçada alternada e nados com braçada simultânea) na capacidade de estabilização pélvica no plano transverso, operacionalizada como assimetria e magnitude da queda pélvica. Os resultados demonstraram que, ao contrário da hipótese assumida, no presente estudo, o estilo de nado praticado não influenciou na assimetria da queda pélvica. Por outro lado, a magnitude da queda pélvica foi maior nos atletas que praticavam braçada alternada quando comparados aos que praticavam braçada simultânea. Esse fato sugere que, apesar da ocorrência de prática de movimentos rotatórios de tronco em nadadores de estilos com braçadas alternadas, a capacidade de estabilização pélvica desses atletas está reduzida. Outro fato que deve ser ressaltado foi a presença de maior queda pélvica na terceira repetição em todos os nadadores, quando comparada à primeira e segunda repetição do teste. Essa observação sugere que, apesar da intensa prática dos nados avaliados (pelo menos cinco anos), esses atletas apresentam uma tendência a entrar em fadiga da musculatura estabilizadora lombopélvica com a repetição do movimento. Dessa forma, os estilos de nado e os tipos de demanda impostos sobre os nadadores em treinos e competições podem estar relacionados à capacidade de estabilização pélvica desses atletas.

Apesar da diferença do gesto esportivo nos nados crawl e costas (pernada e braçada alternadas, movimentos rotatórios de pelve e tronco) quando comparado com os nados borboleta e peito (pernada e braçada simultâneas, movimentos de pelve e tronco no plano sagital), não foi encontrada diferença na assimetria de queda pélvica no plano transverso entre esses grupos. Este estudo apresentou como hipótese que a prática dos nados crawl e costas favorece uma maior assimetria de queda pélvica decorrente da maior amplitude de rotação de tronco e pelve quando comparado a prática dos demais nados ${ }^{12,14}$. A maior amplitude de rotação desses nados tornaria os seus praticantes mais susceptíveis a fatores que contribuem com a assimetria de capacidade de estabilização pélvica, como a dominância de membro superior. Evidências sugerem que o membro superior dominante gera maior capacidade de produção de torque ${ }^{15}$. A maior força encontrada no membro superior dominante do nadador contribui para uma melhor transmissão de força para o segmento lombopélvico, o que pode resultar em assimetria no complexo cruzado de oblíquos e, consequentemente, na assimetria de rotação de tronco e pelve durante o nado. Apesar desses fatores contribuírem para a assimetria no movimento lombopélvico, a hipótese de diferença entre os grupos para a variável assimetria não foi confirmada. Isso ocorreu devido à magnitude do movimento pélvico no plano transverso. Psycharakis e Sanders ${ }^{20}$ observaram uma maior magnitude de rotação do tronco do que da pelve, a qual indica que as assimetrias de tronco no plano transverso são maiores do que as de pelve. Dessa forma, apesar da prática de braçada alternada contribuir com assimetrias de rotação do tronco e da pelve, a assimetria pélvica apresenta menor magnitude que a de tronco. Além disso, a possibilidade dos movimentos rotatórios de tronco, apesar dos efeitos da lateralidade descritos, não produzirem movimentos assimétricos deve ser fortemente considerada.

Diferentemente do hipotetizado, os nados de braçada simultânea (peito e borboleta) apresentaram melhor capacidade de estabilização pélvica no plano transverso que os nados de braçada alternada (crawl e costas). Isso indica que a demanda para minimizar o movimento pélvico no plano transverso durante os nados de braçada simultânea é superior à demanda para o controle pélvico nesse plano durante os nados de braçada alternada. Para o melhor desempenho das ondulações dos nados peito e borboleta, é importante que o atleta minimize as forças que atuam na rotação do segmento lombopélvico. A adaptação do tecido musculoesquelético à ação contínua dessas forças² (por exemplo, por meio da melhora da capacidade de gerar torque muscular e aumento do trofismo muscular) aumenta a capacidade de estabilização pélvica desses atletas no plano transverso. Além disso, nos nados de braçada simultânea, principalmente no nado borboleta, ocorre uma grande demanda dos músculos abdominais, extensores da coluna e quadril na realização do movimento ondulatório do corpo ${ }^{3}$. Estudos identificaram maior atividade eletromiográfica desses grupos musculares durante o teste da ponte ${ }^{18,21}$. É importante considerar que o pequeno valor da diferença entre as médias da magnitude de queda pélvica encontrada entre grupos poderia indicar pouca aplicabilidade clínica dos resultados. Entretanto, o valor encontrado representa uma diferença de 14\% entre os grupos. Assim, apesar da diferença entre as médias dos grupos ter sido de apenas 2,33 ${ }^{\circ}$, os resultados demonstram que o grupo de braçada alternada possui menor capacidade de estabilização pélvica do que o grupo de braçada simultânea.

Uma interpretação adicional para os achados deste estudo seria que a menor capacidade de estabilização pélvica nos nadadores de crawl e costas seja uma característica desses estilos. Nessa perspectiva, o tecido musculoesquelético do segmento lombopélvico nos nadadores desses estilos estaria adaptado a permitir uma maior deformação no plano transverso. Essa maior deformação contribuiria com o desempenho ótimo do nado no que diz respeito às suas características biomecânicas, uma vez que, a cada braçada, o movimento no plano transverso produzido alonga músculos e outros tecidos moles (como fáscia) do tronco, armazenando energia, que será reaproveitada na próxima braçada. Esse mecanismo de movimentos de tronco e pelve no plano transverso no reaproveitamento de energia é similar a outros esportes, como durante o chute do futebol ${ }^{22}$. Apesar de característico desses estilos, a maior magnitude de movimento no plano transverso contribui com maior sobrecarga na coluna lombar e predispor esses atletas a lesão desse segmento ${ }^{23}$. Dessa forma, atletas que praticam braçada simultânea lidam com forças que geram adaptação tecidual suficiente para garantir uma melhor capacidade de estabilização pélvica no plano transverso.

Os atletas apresentaram maior magnitude de queda pélvica na terceira repetição do teste da ponte com extensão unilateral do joelho quando comparada a primeira e segunda repetição, independente da braçada praticada. O aumento da queda pélvica ao longo do teste é um indicador de menor capacidade da musculatura lombopélvica para sustentar o alinhamento pélvico no plano transverso, ou seja, menor capacidade de estabilização pélvica ao longo do teste. Outro estudo ${ }^{18}$ já utilizou esse teste 
como evolução da posição de ponte em supino com objetivo de avaliar a resistência muscular em pacientes com dor lombar. Dessa forma, o teste da ponte com extensão unilateral do joelho pode ser uma importante ferramenta clínica na avaliação da capacidade de estabilização pélvica no plano transverso, em situações nas quais ocorre fadiga muscular. Para isso, sugere-se a execução de pelo menos três repetições desse teste.

Os achados de redução da capacidade de estabilização pélvica ao longo das repetições do teste sugere que os atletas apresentariam quadro similar ao percorrerem longas distâncias em treinos ou em competições. A fadiga da musculatura lombopélvica é um dos fatores contribuintes para o surgimento de lesões na coluna lombar, comum em nadadores ${ }^{24,25}$. Em suporte a isso, Tate et al. ${ }^{25}$, em um estudo epidemiológico com nadadores universitários norte-americanos e nadadoras de diferentes idades, identificaram uma associação entre volume de treinamento realizado e a incidência de lesões. Nessa perspectiva, um nadador que diminua a capacidade de lidar com forças no plano transverso ao longo do treino ou competição, fica exposto a maiores forças de torção sobre a coluna lombar e assim, pode desenvolver uma lombalgia. Além disso, esse tipo de força está mais relacionado a processos degenerativos na coluna lombar, o que também contribui com o surgimento de dores lombares ao longo do tempo $23,26,27$.

Além de lesões em segmentos próximos, a fadiga da musculatura lombopélvica também contribui com a lesão em segmentos distantes. A menor capacidade de estabilização pélvica, resultante da fadiga muscular, leva a uma transmissão inadequada de forças para a cintura escapular ${ }^{3}$ e reduz o desempenho dos músculos que agem sobre o ombro durante a braçada. Devido à ligação mecânica entre os músculos ${ }^{8}$, uma menor capacidade dos músculos do tronco e da pelve está relacionada com o surgimento de patologias no ombro, quadro comum em nadadores $24,28,29$. Essa hipótese é reforçada pela evidência de que nadadores fundistas e nadadores com elevado volume de treino apresentam maior número de lesões no ombro ${ }^{30}$. Assim, programas preventivos devem considerar a relação entre a resistência da musculatura lombopélvica com a capacidade do ombro lidar com a alta demanda da prática da natação. Ressalta-se que apesar dos resultados sugerirem que atletas de natação com baixa

\section{REFERÊNCIAS}

1. Fonseca ST, Ocarino JM, Silva PLP, Aquino CF. Integration of stresses and their relationship to the kinetic chain. In: Magee DJ, Zachazewski JE, Quillen WS, editors. Scientific foundations and principles of practice in musculoskeletal rehabilitation. St. Louis: Saunders Elsevier; 2007. p. 476-86.

2. Mueller MJ, Maluf KS. Tissue adaptation to physical stress: a proposed "physical stress theory" to guide physical therapist practice, education, and research. PhysTher. 2002;82(4):383-403.

3. Pink MM, Edelman GT, Mark R, Rodeo SA. Applied Biomechanics of Swimming. In: Magee DJ, Manske RC, Zachazewski JE, Quillen WS, editors. Athletic and sport issues in musculoskeletal rehabilitation. St. Louis: Saunders Elsevier; 2011. p. 331-49.

4. KiblerWB, Press J, Sciascia A. The role of core stability in athletic function. Sports Med. 2006;36(3):189-98.

5. Deschodt VJ, Arsac LM, Rouard AH. Relative contribution of arms and legs in humans to propulsion in 25-m sprint front-crawl swimming. Eur J Appl Physiol Occup Physiol. 1999;80(3):192-9.

6. Rubin BD, Kibler WB. Fundamental principles of shoulder rehabilitation: conservative to postoperative management. Arthroscopy. 2002;18(9 Suppl 2):29-39.

7. Levin SM. A different approach to the mechanics of the human pelvis: tensegrity. In: Vleeming A Mooney V, Snijders CJ, Dorman TA, Stoeckart R, editors. Movement, stability and low back pain. Edinburgh: Churchill Livingstone; 1997. p. 157-67.

8. Myers TW. The spiral line. In: Myers TW, editor. Anatomy trains - myofascial meridians for manual and movement therapists. Edinburgh: Churchill Livingstone; 2001. p. 139-58.

9. Pool-Goudzwaard AL, Vleeming A, Stoeckart R, Snijders CJ, Mens JM. Insufficient lumbopelvic stability: a clinical, anatomical and biomechanical approach to 'a-specific' low back pain. Man Ther. 1998;3(1):12-20

10. Carvalhais VOC, Ocarino JM, Araújo VL, Souza TR, Silva PLP, Fonseca ST. Myofascial force transmission between the latissimus dorsi and gluteus maximus muscles: an in vivo experiment. J Biomech. 2013;46(5):1003-7.

11. Kibler WB. The role of the scapula in athletic shoulder function. Am J Sports Med. 1998;26(2):325-37.

12. Tovin BJ. Prevention and treatment of swimmer's shoulder. N Am J Sports Phys Ther. 2006;1 (4):166-75.

13. Yanai T. Buoyancy is the primary source of generating bodyrollin front-crawl swimming. J Biomech. 2004;37(5):605-12

14. Secchi LLB, Muratt MD, Andrade NVS, Greve JMD. Dinamometriaisocinética de tronco em nadadores de diferentes estilos. Acta Ortop Bras. 2010;18(5):295-7.

15. Olivier N, Quintin G, Rogez J. The high level swimmer articular shoulder complex. Ann Readap Med Phys. 2008;51(5):342-7.

16. Michaud TC. Abnormal Motion during the Gait Cycle. In: Michaud TC. Foot orthoses and other forms of conservative foot care. Baltimore: Williams \& Wilkins; 1993. p. 57-180. capacidade de estabilização pélvica devido à fadiga estariam sujeitos ao desenvolvimento de lesões musculoesqueléticas, estudos são necessários para investigar o impacto de alterações do alinhamento pélvico no plano transverso na ocorrência de disfunções em nadadores.

Este artigo apresenta algumas limitações. A ausência de diferença na assimetria de queda pélvica entre os grupos seria influenciada por possíveis programas de exercícios compensatórios por parte dos treinadores, uma vez que este estudo não monitorou as características do treinamento dos atletas. Dessa forma, sugere-se que futuros estudos considerem essa influência na capacidade de estabilização pélvica. Além disso, a utilização de um teste fora da piscina para avaliar a capacidade de estabilização pélvica de nadadores poderia não ser apropriado. Contudo, a escolha do teste levou em consideração não só as suas propriedades clinimétricas adequadas e sua fácil aplicação, mas também sua aplicabilidade para indexar a capacidade de indivíduos de estabilizar o tronco e pelve em contextos gerais.

A partir dos resultados encontrados, demonstramos que nadadores que praticam braçada alternada ou simultânea não diferem quanto à assimetria de queda pélvica. Contudo, nadadores que praticam estilos com braçada alternada apresentam maior magnitude da queda pélvica, o que estaria relacionado às adaptações teciduais do gesto esportivo.

\section{CONCLUSÃO}

As diferenças entre as repetições do teste indicam que a partir da terceira repetição os nadadores apresentam uma menor capacidade de estabilização pélvica no plano transverso. Esse fato indica que os músculos lombopélvicos estão sujeitos à fadiga, o que é um fator de risco para o desenvolvimento de disfunções. Assim, programas preventivos com nadadores devem considerar as adaptações geradas pelo gesto esportivo e a menor capacidade de estabilização pélvica que esses atletas apresentam ao longo do tempo.

Todos os autores declararam não haver qualquer potencial conflito de interesses referente a este artigo.

17. Leetun DT, Ireland ML, Willson JD, Ballantyne BT, Davis IM. Core stability measures as risk factors for lower extremity injury in athletes. Med Sci Sports Exerc. 2004;36(6):926-34.

18. Schellenberg KL, Lang JM, Burnham RS. A clinical tool for office assessment of lumbar spine stabilization endurance: prone and supine bridge maneuvers. Am J Phys Med Rehabil. 2007;86(5):380-6.

19. Andrade JA, Fiqueiredo LC, Santos TRT, Paula ACV, Bittencourt NFN, Fonseca ST. Confiabilidade da mensuração do alinhamento pélvico no plano transverso durante o teste da ponte com extensão unilateral do joelho. Rev Bras Fisioter. 2012;16(4):268-74.

20. Psycharakis SG, Sanders RH. Shoulder and hip roll changes during 200-m front craw/ swimming. Med Sci Sports Exerc. 2008:40(12):2129-36

21. Stevens VK, Bouche KG, Mahieu NN, Coorevits PL, Vanderstraeten GG, Danneels LA. Trunk muscle activity in healthy subjects during bridging stabilization exercises. BMC Musculoskelet Disord. 2006;7:75-82.

22. Fonseca ST, Souza TR, Ocarino JM, Gonçalves GP, Bittencourt NF. Applied biomechanics of soccer. In: Magee DJ, Manske RC, Zachazewski JE, Quillen WS, editors. Athletic and sport issues in musculoskeletal rehabilitation. St. Louis: Elsevier Saunders; 2011. p. 287-306.

23. Farfan HF, Cossette JW, Robertson GH, Wells RV, Kraus H. The effects of torsion on the lumbar intervertebral joints: the role of torsion in the production of disc degeneration. J Bone Joint Surg Am. 1970;52(3):468-97.

24. Osawa CC, Andries Júnior O. Aspectos de saúde da equipe de natação da UNICAMP. Motriz. 2006;12(2):149-58.

25. Tate A, Turner GN, Knab SE, Jorgensen C, Strittmatter A, Michener LA. Risk factors associated with shoulder pain and disability across the lifespan of competitive swimmers. J Athl Train. 2012;47(2):149-58.

26. Blankenbaker DG, Haughton VM, Rogers BP, Meyerand ME, Fine JP. Axial rotation of the lumbar spinal motion segments correlated with concordant pain on discography: a preliminary study. AJR Am J Roentgenol. 2006;186(3):795-9.

27. Kaneoka K, Shimizu K, Hangai M, Okuwaki T, Mamizuka N, Sakane M, et al. Lumbar intervertebral disk degeneration in elite competitive swimmers: a case control study. Am J Sports Med 2007;35(8):1341-5.

28. Weldon EJ 3rd, Richardson AB. Upper extremity overuse injuries in swimming. A discussion of swimmer's shoulder. Clin Sports Med. 2001;20(3):423-38

29. Venâncio BO, Tacani PM, Deliberato PCP. Prevalência de dor nos nadadores de São Caetano do Sul. Rev Bras Med Esporte. 2012;18(6):394-9.

30. Aguiar PRC, Bastos FN, Júnior JN, Vanderlei LCM, Pastre CM. Lesões Desportivas na Natação. Rev Bras Med Esporte. 2010;16(4):273-7. 